\title{
Lectura Digital: Incidencia de la práctica digital en los hábitos de lectura Proyecto de Investigación \\ $\mathrm{N}^{\circ} 6.1$ \\ Equipo de Investigación ${ }^{(1)}$ \\ Ivana Mihal
}

por Facultad de Diseño y Comunicación, Universidad de Palermo (ARG)

\begin{abstract}
Resumen: El Proyecto de Investigación 6.1 presenta distintas aproximaciones que atraviesan la convergencia pedagógica-digital particularmente en el universo de los libros, y lecturas y sus cruces con el diseño. A través de miradas distintas que abarcan desde ámbitos concretos del diseño, la comunicación y la edición y disciplinas afines como la antropología, la sociología, la historia, la literatura, la bibliotecología, y las ciencias de la educación, entre otras, vinculadas con problemáticas de lectura y escritura, y a aquellas más ligadas a la producción y circulación de libros en distintos soportes, se apunta a introducir un conjunto de tópicos que constituyen desafíos a la hora de indagar en dicha convergencia.
\end{abstract}

Palabras clave: Diseño digital - Comunicación - Edición - Convergencia pedagógica Desafíos - Libros - Lecturas

[Resúmenes en inglés y portugués en la página 170]

${ }^{(1)}$ Los CVs del Equipo de Investigación pueden consultarse en el Capítulo Directores de Líneas y Coordinadores de Proyectos de esta misma Edición.

\section{Acerca del Proyecto 6.1}

Lectura Digital:

Incidencia de la práctica digital en los hábitos de lectura

El Proyecto 6.1 Lectura Digital: Incidencia de la práctica digital en los hábitos de lectura, reúne diversas perspectivas, en un estudio interdisciplinario en el marco de las políticas públicas, siendo que el mundo digital ha tenido un significativo desarrollo en los últimos tiempos, que incide sobre casi todas las actividades del ser humano, por tanto también, en las prácticas de lectura. La industria del libro y las nuevas y/o renovadas configuraciones en los modos de leer ameritan un acercamiento esta particular problemática, centrando el 
campo específico de los estudios en la cultura y la creatividad, para que sea posible identificar e indagar los alcances y desafíos que se plantean.

Avanza en una investigación en la que participan investigadores, provenientes de distintas disciplinas, como comunicación, diseño, historia, antropología, sociología, ciencias de la educación, entre otras carreras afines, con la coordinación de Ivana Mihal (UP), con el fin de abordar las transformaciones socioculturales en las prácticas, en las concepciones, y en las políticas que se entretejen a la hora de pensar la problemática, e Impactar en la formación de los Diseñadores Gráficos, puntualmente a través de los contenidos del Diseño Editorial y la Lectura digital.

Las reflexiones y los resultados obtenidos en el Proyecto 6.1 Lectura Digital: Incidencia de la práctica digital en los hábitos de lectura, se continúan desarrollando en los Proyectos 6.2 Edición universitaria y políticas editoriales como objeto de análisis, y 6.3 Producción, Circulación y Comunicación del conocimiento en Instituciones de Educación Superior: Políticas Editoriales, en el marco de la misma Línea de Investigación y bajo la misma Directora Ivana Mihal.

Sus principales objetivos son:

- Estudiar los ámbitos concretos de abordaje del diseño, la comunicación y la edición de libros digitales en relación con las problemáticas de lectura y escritura, tanto como la producción y circulación de libros en distintos soportes;

- Analizar la incidencia del mundo digital en las prácticas de lectura y en las producciones específicas de la industria editorial digitalizada;

- Explorar los entrecruzamientos de libros impresos y lo digital

- Estudiar, desde distintas perspectivas, la incidencia de lo digital en el diálogo libros y lecturas con el diseño y la comunicación, junto con los procesos que involucran estos temas;

- Ahondar en los consumos culturales de la sociedad contemporánea, mediante el análisis del sector editorial de libros y las distintas maneras de leer en la actualidad

\section{Acerca de la Línea 6}

Convergencia pedagógica digital: Miradas interdisciplinarias dirigida por Ivana Mihal se desarrolla de manera ininterrumpida desde 2015 en la Facultad de Diseño y Comunicación (UP, Argentina), e incluye hasta el momento tres proyectos finalizados el 6.1 Lectura Digital: Incidencia de la práctica digital en los hábitos de lectura coordinado por Ivana Mihal, el 6.2 Edición universitaria y políticas editoriales como objeto de análisis coordinado por Ivana Mihal (UP) y Daniela Szpilbarg (Instituto de Desarrollo Económico y Social, IDES-CONICET, Argentina), y el 6.3 Producción, Circulación y Comunicación del conocimiento en Instituciones de Educación Superior: Políticas Editoriales, coordinado por Ivana Mihal (Universidad de Palermo, y Laboratorio de Investigación en Ciencias Humanas de la UNSAM-CONICET, Argentina), Ana Elisa Ribeiro (Centro Federal de Educacao Tecnológica de Minas Gerais-CEFET-MG, Brasil) y Daniela Szpilbarg (Instituto de Desarrollo Económico y Social, IDES-CONICET, Argentina). 


\section{Mapa de Áreas y Proyectos}

El Proyecto 6.1 se vincula con todas las carreras de grado y posgrado correspondientes a la Facultad de Diseño y Comunicación dado que impacta en la incorporación de la investigación en la lógica de la docencia universitaria, y específicamente de manera fundamental con la carrera de Diseño gráfico y Comunicación digital. Además los proyectos de la Línea de Investigación están vinculados también con los de la Línea de Investigación No9 Enseñar Disciplinas Proyectuales.

\section{Productos y Resultados}

\section{a)- Publicaciones}

Cuaderno del Centro de Estudios de Diseño y Comunicación No72. (2019) Convergencia pedagógica digital: libros, lecturas y diseño. Facultad de Diseño y Comunicación Año XIX, Mayo 2019, Buenos Aires, Argentina. ISSN: 1668-0227. Esta publicación documenta y comunica los resultados alcanzados en el proyecto de investigación 6.1 Lectura Digital (Finalizado), y a continuación se detallan los autores y artículos contenidos en ella:

Aguerre, Natalia (2019) Arte y Medios: Narrativa transmedia y el translector (Pp. 17-32)

Albarello, Francisco (2019) El lector en la encrucijada: la lectura/navegación en las pantallas digitales (Pp. 33-43)

Bombini, Gustavo (2019) Didáctica de la lectura y la escritura y multimodalidad (Pp. 71-79)

Calabre, Lia (2019) Planos de livro e leitura em tempos da cultura digital (Pp. 109-120)

Franco, María del Carmen Rosas (2019) Nuevos soportes, nuevos modos de leer. La narrativa en la Literatura infantil y juvenil digital (Pp. 45-58)

Sorrentino, Florencia Lila (2019) Instantáneas: la lectura en los tiempos que corren (Pp. 59-70)

Landau, Mariana (2019) Los discursos sobre tecnologías y educación en la esfera pública (Pp. 81-93)

Ligia Medeiros Ana, Olinto Gilda (2019) O impacto da tecnologia de informação e comunicação nas bibliotecas públicas: envolvimento comunitário, criatividade e inovaçã (Pp. 121-134)

Pereyra, Eduardo (2019) Juventudes y TIC: Estados locales frente al abordaje de la promoción de la lectura (Pp. 135-150)

Pini, Mónica (2019) Políticas de alfabetización digital. Educación e inclusión (Pp. 95-107)

Mihal, Ivana (2019) Prólogo. Convergencia pedagógica-digital: libros, lecturas y diseño (Pp. 11-15)

Szpilbarg, Daniela (2019) Configuraciones emergentes de circulación y lectura en el entorno digital: el caso de Bajalibros.com (Pp. 151-165) 
Actas de Diseño No27 (2018) Semana Internacional del Diseño en Palermo. Facultad de Diseño y Comunicación UP. En esta publicación se documentan las ponencias de la Comisión Convergencia pedagógico digital: Proyecto 6.1 Lectura Digital correspondientes a la presentación del Cuaderno 72 durante el III Coloquio de Investigación y Desarrollo en Diseño (Pp. 48-49).

Actas de Diseño No24. (2018) XII Encuentro Latinoamericano de Diseño "Diseño en Palermo". Foro de Escuelas de Diseño - XI Plenario 2017, VIII Congreso Latinoamericano de Enseñanza del Diseño. II Coloquio de Investigación y Desarrollo en Diseño Latino. Coloquio de Investigación. Julio 2017, Buenos Aires, Argentina. En esta publicación se documentan las ponencias presentadas en la Comisión de la Línea de Investigación No6 Convergencia pedagógico digital en el II Coloquio de Investigación y Desarrollo en Diseño (Pp. 37-38).

Escritos en la Facultad No122. (2016) Articulación entre Investigación y Posgrados, Edición 1. Doctorado en Diseño. Maestría en Gestión del Diseño. Programa de Investigación y Desarrollo en Diseño Latino. Línea de Investigación N6: Convergencia pedagógica digital. Miradas interdisciplinarias. ISSN: 1669-2306. ISSN (En línea): 2591-3743. En esta publicación la Directora Ivana Mihal presenta la línea de investigación No6 Convergencia pedagógico digital y anuncia el comienzo del Proyecto 6.1 Lectura Digital (Pp.11-12).

\section{b)- Congresos / Coloquios / Plenarios}

$1^{\circ}$ Plenario de Directores de Investigación DC, 13 de octubre - 2017. Es este primer plenario los Directores de Líneas y Proyectos de Investigación presentaron a sus pares y al conjunto del Programa de Investigación de la Facultad de Diseño y Comunicación, los resultados obtenidos y/o en proceso (publicaciones y acuerdos), junto con los Proyectos. La Directora de la Línea de Investigación N6 presentó a sus pares, los principales avances del Proyecto 6.1 Lectura Digital (Finalizado).

II Coloquio de Investigación y Desarrollo en Diseño Latino. Universidad de Palermo, 31 de julio de 2017. VIII Congreso Latinoamericano de Enseñanza del Diseño. Semana Internacional del Diseño en Palermo.

En la comisión Convergencia pedagógica digital: miradas Interdisciplinarias, se presentaron las reflexiones y avances del proyecto 6.1 Lectura Digital (Finalizado) expusieron: Natalia Aguerre, Francisco Albarello, Gustavo Bombin, Lia Calabre, María del Carmen Rosas Franco, Ana Ligia Silva Medeiros, Gilda Olinto, Florencia Lila Sorrentino y Daniela Szpilbarg.

A continuación se detallan las ponencias presentadas en esta Comisión coordinada por la Directora del proyecto Ivana Mihal:

Aguerre Natalia

Arte y Medios: Narrativa transmedia y el translector 


\begin{abstract}
Albarello Francisco
El lector en la encrucijada: la lectura/navegación en las pantallas digitales

Bombini Gustavo

Didáctica de la lectura y la escritura y multimodalidad

Calabre Lia

Planes de libros y la lectura en los tiempos de cultura digital

Rosas Franco María del Carmen

Nuevos soportes, nuevos modos de leer. La narrativa en la literatura infantil y juvenil digital

Silva Medeiros Ana Ligia, Olinto Gilda

O impacto da tecnologia de informação e comunicação nas bibliotecas públicas: envolvimento comunitário, criatividade e inovação

Lila Sorrentino Florencia

Instantáneas: la lectura en los tiempos que corren

Szpilbarg Daniela

Configuraciones emergentes de circulación y lectura en el entorno digital: el caso de Bajalibros.com
\end{abstract}

I Coloquio de Investigación y Desarrollo en Diseño Latino. Universidad de Palermo, 26 de Octubre de 2016. Se presentó la Línea de Investigación N6: Convergencia Pedagógica Digital. Miradas interdisciplinarias, como una de las líneas que integran el Programa de Investigación en Diseño de la Facultad de Diseño y Comunicación.

Mihal Ivana. (Argentina) Presentación de la Línea de Investigación N6: Convergencia pedagógica digital: miradas interdisciplinarias. Facultad de Diseño y Comunicación, Universidad de Palermo.

\title{
c)- Formación de Posgrado e Impacto curricular
}

La Directora Ivana Mihal es Docente de Posgrado en Diseño de la Facultad de Diseño y Comunicación: Maestría en Gestión del Diseño y Doctorado en Diseño, e incorpora los contenidos de su investigación a sus asignaturas.

\section{Evaluación Externa}

El Proyecto 6.1 Lectura Digital: Incidencia de la práctica digital en los hábitos de lectura, cuyos resultados fueron publicados en el Cuaderno No72, fue evaluado exitosamente por los Evaluadores Martin Boy y Julio Enrique Putallaz en 2019. 


\begin{abstract}
Research Project 6.1 presents different approaches that cross the pedagogicaldigital convergence, particularly in the universe of books, and readings and their intersections with design. Through different perspectives that range from specific areas of design, communication and publishing and related disciplines such as anthropology, sociology, history, literature, library science, and educational sciences, among others, linked to problems of reading and writing, and those more linked to the production and circulation of books in different media, aims to introduce a set of topics that constitute challenges when investigating this convergence.
\end{abstract}

Keywords: Digital design - Communication - Editing - Pedagogical convergence - Challenges - Books - Readings

Resumo: O Projeto de Pesquisa 6.1 apresenta diferentes abordagens que cruzam a convergência pedagógico-digital, principalmente no universo dos livros, e das leituras e seus cruzamentos com o design. Através de diferentes perspectivas que vão desde áreas específicas de design, comunicação e publicação e disciplinas afins, como antropologia, sociologia, história, literatura, biblioteconomia e ciências da educação, entre outras, vinculadas a problemas da leitura e da escrita, e aquelas mais vinculadas à produção e circulação de livros em diferentes meios, visa apresentar um conjunto de temas que constituem desafios para a investigação dessa convergência.

Palavras chave: Design digital - Comunicação - Edição - Convergência pedagógica - Desafios - Livros - Leituras

[Las traducciones de los resúmenes fueron realizadas a través de traductor automático] 